\title{
Subgrid-Modelling in LES of Compressible Flow
}

\author{
BERT VREMAN, BERNARD GEURTS and HANS KUERTEN \\ Department of Applied Mathematics, University of Twente, P.O. Box 217, 7500 AE Enschede, \\ The Netherlands
}

Received 11 July 1994; accepted in revised form 7 February 1995

\begin{abstract}
Subgrid-models for Large Eddy Simulation (LES) of compressible turbulent flow are tested for the three-dimensional mixing layer. For the turbulent stress tensor the recently developed dynamic mixed model yields reasonable results. A priori estimates of the subgrid-terms in the filtered energy equation show that the usually neglected pressure-dilatation and turbulent dissipation rate are as large as the commonly retained pressure-velocity subgrid-term. Models for all these terms are proposed: a similarity model for the pressure-dilatation, similarity and $k$-dependent models for the turbulent dissipation rate and a dynamic mixed model for the pressure-velocity subgrid-term. Actual LES demonstrates that for a low Mach number all subgrid-terms in the energy equation can be neglected, while for a moderate Mach number the effect of the modelled turbulent dissipation rate is larger than the combined effect of the other modelled subgrid-terms in the filtered energy equation.
\end{abstract}

Key words: large eddy simulation, compressible mixing layer

\section{Introduction}

Most turbulent flows contain too many scales for a Direct Numerical Simulation (DNS) of the Navier-Stokes equations. A Large Eddy Simulation (LES) tackles this problem by solving only the large scales, while the effect of the small (subgrid-) scales is modelled (Rogallo and Moin [1]). Considerable effort has been directed towards the development of subgrid-models for LES of incompressible flows. Many of these subgrid-models are of the eddy-viscosity type. An eddy-viscosity model becoming increasingly popular is the dynamical formulation of the Smagorinsky model by Germany [2]. An example of a successful non-eddy-viscosity model is the mixed model by Bardina et al. [3], in which Smagorinsky's model has been supplemented with a similarity idea. Recently Zang et al. [4] proposed a dynamical formulation of the mixed model.

Since the work of Yoshizawa [5], who generalised the Smagorinsky model, an increasing amount of research has been conducted on LES of compressible flow. Erlebacher et al. [6] have extended the standard mixed model to compressible isotropic turbulence, while Moin et al. [7] formulated the dynamic model for compressible LES, which has been applied to e.g. the compressible boundary layer (El-Hady et al. [8]). In this paper we extend the dynamic mixed model to compressible flow. Subgrid-modelling in compressible LES requires modelling of the subgrid-terms in the energy equation, or in equivalent formulations, the pressure or temperature equation. We will show that two of these terms, the pressure-dilatation 
and the turbulent dissipation rate, which are generally neglected in compressible LES, are as large as the usually retained subgrid-terms. Therefore models for these terms are proposed and tested at low and moderate Mach numbers. Simulations show that in particular the turbulent dissipation rate cannot always be omitted. Compressible turbulence has more extensively been studied using the ensemble averaging approach (e.g. [9-11]), instead of the filtering approach in LES. In this case the pressure-dilatation and turbulent dissipation rate have also been found to be important.

This paper is organised as follows. Section 2 presents the filtered Navier-Stokes equations. Section 3 presents several subgrid-models which are tested a priori, using DNS results of the three-dimensional compressible mixing layer. Results for LES with several subgrid-models are shown in Section 4. Finally, Section 5 summarises our findings.

\section{Governing Equations}

For LES of compressible flows the basic governing equations are the filtered Navier-Stokes equations (Geurts et al. [12]),

$$
\begin{aligned}
& \partial_{t} \bar{\rho}+\partial_{j}\left(\bar{\rho} \tilde{u}_{j}\right)=0, \\
& \partial_{t}\left(\bar{\rho} \tilde{u}_{i}\right)+\partial_{j}\left(\bar{\rho} \tilde{u}_{i} \tilde{u}_{j}\right)+\partial_{i} \bar{p}-\partial_{j} \hat{\sigma}_{i j}=-\partial_{j}\left(\bar{\rho} \tau_{i j}\right), \\
& \partial_{t} \hat{e}+\partial_{j}\left((\hat{e}+\bar{p}) \tilde{u}_{j}\right)-\partial_{j}\left(\hat{\sigma}_{i j} \tilde{u}_{i}\right)+\partial_{j} \hat{q}_{j}=-\alpha-\beta-\pi+\varepsilon,
\end{aligned}
$$

where the overbar denotes a filter operation, which commutes with the partial derivatives $\partial_{t}$ and $\partial_{j}$. The second filter, represented by a tilde, refers to the Favre filter operation (Erlebacher et al. [6]), which is defined as $\tilde{u}_{i}=\bar{\rho} \overline{u_{i}} / \bar{\rho}$. Concerning the flow variables, $\rho, \mathbf{u}$ and $p$ are the density, velocity and pressure, respectively. Moreover, $\hat{e}$ is the total energy density of the filtered variables

$$
\hat{e}=\frac{\bar{p}}{\gamma-1}+\frac{1}{2} \bar{\rho} \tilde{u}_{i} \tilde{u}_{i} \text {. }
$$

Unlike the 'bar' and the 'tilde', the 'hat'-symbol does not denote a filter operation but indicates that the quantity is based on filtered variables. Thus $\hat{e}$ refers to the resolved total energy, which is not equal to the filtered total energy. The viscous stress tensor $\hat{\sigma}_{i j}$ is defined as

$$
\hat{\sigma}_{i j}=\frac{\mu(\tilde{T})}{\operatorname{Re}} \hat{S}_{i j} \quad \text { with } \quad \hat{S}_{i j}=\partial_{j} \tilde{u}_{i}+\partial_{i} \tilde{u}_{j}-\frac{2}{3} \delta_{i j} \partial_{k} \tilde{u}_{k},
$$

where the viscosity $\mu(\tilde{T})$ is given by Sutherland's law for air. In addition $\hat{\mathbf{q}}$ represents the heat flux vector, given by

$$
\hat{q}_{j}=-\frac{\mu(\tilde{T})}{(\gamma-1) \operatorname{RePrM}^{2}} \partial_{j} \tilde{T}
$$


The filtered temperature $\tilde{T}$ is obtained from the equation of state

$$
\tilde{T}=\gamma \mathbf{M}^{2} \frac{\bar{p}}{\bar{\rho}} .
$$

The ratio of the specific heats, $\gamma$ and the Prandtl number Pr are given the values 1.4 and 1 respectively, while the values of the Reynolds number Re and the Mach number $\mathrm{M}$ will be specified below.

The right-hand sides of the equations (1-3) contain the so-called subgrid-terms, which represent the effect of the unresolved subgrid-scales. The turbulent stress tensor $\tau_{i j}$ is defined as

$$
\tau_{i j}=\widetilde{u_{i} u_{j}}-\tilde{u}_{i} \tilde{u}_{j},
$$

while the subgrid-terms in the filtered energy equation have the following form:

$$
\begin{aligned}
\alpha & =\tilde{u}_{i} \partial_{j}\left(\bar{\rho} \tau_{i j}\right), \\
\beta & =\partial_{j}\left(\overline{p u_{j}}-\bar{p} \tilde{u}_{j}\right) /(\gamma-1), \\
\pi & =\overline{p \partial_{j} u_{j}}-\bar{p} \partial_{j} \tilde{u}_{j}, \\
\varepsilon & =\overline{\sigma_{i j} \partial_{j} u_{i}}-\bar{\sigma}_{i j} \partial_{j} \tilde{u}_{i} .
\end{aligned}
$$

The term $\alpha$ is the turbulent stress on the scalar level. The definition of $\hat{e}$ (equation (4)) implies that equation (3) is the sum of the filtered pressure equation divided by $\gamma-1$ and the equation for the resolved kinetic energy. The equation for the resolved kinetic energy contains the term $\alpha$. Consequently, equation (3) contains this term as well, while $\alpha$ does not appear when equation (3) is replaced by the filtered pressure or temperature equation. Furthermore, $\beta$ is the pressure-velocity subgrid-term, $\pi$ is the pressure-dilatation and $\varepsilon$ is the subgrid-scale turbulent dissipation rate. In equation (12) $\sigma_{i j}$ denotes the viscous stress tensor based on the unfiltered velocity and temperature. Subgrid-terms resulting from nonlinearities in the viscous terms (e.g. $\left.\partial_{j}\left(\hat{\sigma}_{i j}-\bar{\sigma}_{i j}\right)\right)$ and in the heat-flux term, have been omitted in the filtered equations (1-3). When compared to the other subgrid-terms, these contributions are negligible in the cases considered here, as has been verified with a priori tests. In LES of compressible flow (e.g. [6-8]), $\pi$ and $\varepsilon$ have not been modelled. In particular the inclusion of $\varepsilon$ improves the results for a moderate Mach number.

\section{A Priori Tests}

In this section we present a priori tests for subgrid-models, using the results of Direct Numerical Simulations of the three-dimensional temporal compressible mixing layer. A priori tests of subgrid-models are certainly of some value, but need a careful interpretation [13, 14]. In order to draw conclusions about the 
performance of subgrid-models, investigation of the behaviour of subgrid-models in actual simulations (a posteriori tests) is indispensable. Results of a posteriori tests will be presented in Section 4.

\subsection{FILTERING THE DNS RESULTS}

In order to perform Direct Numerical Simulations of the temporal mixing layer we solve the unfiltered Navier-Stokes equations in a rectangular geometry $[0, L] \times$ $[-H / 2, H / 2] \times[0, L]$. Periodic boundary conditions are imposed in the $x_{1}$ - and $x_{3^{-}}$ direction, while in the normal $x_{2}$-direction the boundaries are free slip walls, i.e. the normal velocity and the normal derivatives of the density, pressure and tangential velocity are zero. The basic initial velocity profile is the hyperbolic tangent profile $u=\tanh \left(x_{2}\right)$. The initial mean temperature profile is obtained from the BusemannCrocco Law (Ragab and Wu [15]) and the initial pressure distribution is uniform. Superimposed on the mean profile we put a three-dimensional large amplitude perturbation, as described by Sandham and Reynolds [16]. The equations are nondimensionalised with half the initial vorticity thickness and the initial upper stream values of the density, velocity, temperature and viscosity. In this paper we use $\operatorname{Re}$ $=50$ (cf. Comte et al. [17]), based on half the initial vorticity thickness, while the convective Mach number $\mathrm{M}$ is equal to 0.2 or 0.6 . Moreover the length $L$ of the domain is set equal to four times the wavelength of the most unstable mode according to linear stability theory, which implies $L=59$ and $L=68$ for the cases $\mathrm{M}=0.2$ and $\mathrm{M}=0.6$, respectively. In both cases we choose $H=59$.

For the DNS, the evolution equations are discretized on a non-staggered uniform grid with $128^{3}$ cells, using central finite differences. The convective terms are discretized with a robust fourth-order accurate method, which approximates e.g. $\partial_{1} f$ as

$$
\begin{aligned}
\left(\partial_{1} f\right)_{i, j, k} & \approx\left(-s_{i+2, j, k}+8 s_{i+1, j, k}-8 s_{i-1, j, k}+s_{i-2, j, k}\right) / 12 h_{1}, \\
s_{i, j, k} & =\left(-g_{i, j-2, k}+4 g_{i, j-1, k}+10 g_{i, j, k}+4 g_{i, j+1, k}-g_{i, j+2, k}\right) / 16, \\
g_{i, j, k} & =\left(-f_{i, j, k-2}+4 f_{i, j, k-1}+10 f_{i, j, k}+4 f_{i, j, k+1}-f_{i, j, k+2}\right) / 16,
\end{aligned}
$$

where $h_{1}$ is the grid-spacing in the $x_{1}$-direction. For the viscous terms a secondorder accurate method is used, which has been described by Kuerten [18]. The explicit time integration is performed with a compact-storage second-order accurate four-stage Runge-Kutta method. To validate the numerical method and to check its accuracy comparisons with linear stability theory have been made. It appears that the growth-rates of the dominant two- and three-dimensional modes are predicted with an error of about one per cent. Furthermore, the results of simulations using a $192^{3}$-grid show that the $128^{3}$-results are sufficiently accurate. The DNS demonstrates the roll-up of the fundamental instability and successive pairings. After the first pairing $(t=40)$ the flow has become highly three-dimensional. Another pairing $(t=80)$, yields a single roller in which the flow exhibits a complex 
structure, with many regions of positive spanwise vorticity. This structure is a result of the transition to turbulence which has been triggered by the pairing process at $t=40$ (Moser and Rogers [19]). The simulations are stopped at $t=100$, at which time a single well-developed roller remains. At this time the transitional regime has already been passed, many small scales have been formed by the mixing transition process and the turbulence in the flow is fully developed. The Reynolds number based on half the vorticity thickness attains values around 600 near the end of the simulation. The tests in this paper are performed at moderate Reynolds numbers, because DNS of the compressible mixing layer at far higher Reynolds numbers is not yet possible. However, for similarity, dynamic and dynamic mixed models $a$ priori tests of subgrid-models have been performed using data from measurements in a turbulent jet at high Reynolds number [20]. Since in this references similar values of the correlations were found as reported for DNS at lower Reynolds numbers, the new results in this paper are expected to be also applicable to flows at higher Reynolds numbers.

In the following results of a priori tests of subgrid-models are presented, using the DNS at $\mathrm{M}=0.2$ and $\mathrm{M}=0.6$. The subgrid-terms introduced in the previous section are calculated according to their definitions. For this purpose we employ the box filter in physical space with the trapezoidal rule. The filter widths $\Delta_{1}, \Delta_{2}$ and $\Delta_{3}$, corresponding to the three coordinate directions, are equal to $L / 16, H / 16$ and $L / 16$, respectively. The following step is to compare the subgrid-terms with model-predictions. In some model formulations a single filter width is needed, which is defined by $\Delta=\left(\Delta_{1} \Delta_{2} \Delta_{3}\right)^{(1 / 3)}$. All a priori tests presented here are conducted using the turbulent fields at $t=80$. Conclusions drawn from the results at this time are typical for all other times past the roll-up at $t=20$.

\subsection{SUBGRID-MODELS FOR THE MOMENTUM EQUATION}

Three subgrid-models for the turbulent stress $\tau_{i j}$ are investigated: the scale similarity model, the dynamic subgrid-model and the dynamic mixed model. In each case we consider the divergences of the turbulent stress as they appear in the momentum equations, i.e. a priori tests on the vector level are performed.

The first model adopts the resolved part of the turbulent stress as a subgridmodel:

$$
\tau_{i j}^{(1)}=\widetilde{\tilde{u}_{i} \tilde{u}_{j}}-\tilde{\tilde{u}}_{i} \tilde{\tilde{u}}_{j} .
$$

This model is obtained if the so-called Leonard term and Bardina's model for the cross-terms (Bardina et al. [3]) are combined. Although this model does not contain an eddy-viscosity and is not purely dissipative, it has dissipation in it and thus takes into account the energy transfer from resolved to subgrid-scales, as can be inferred from literature [20] and the results in Section 4. Figure 1 shows the correlations of several subgrid-models with the exact turbulent stress in parallel planes at constant $x_{2}$. It appears that with the scale similarity model an excellent correlation 


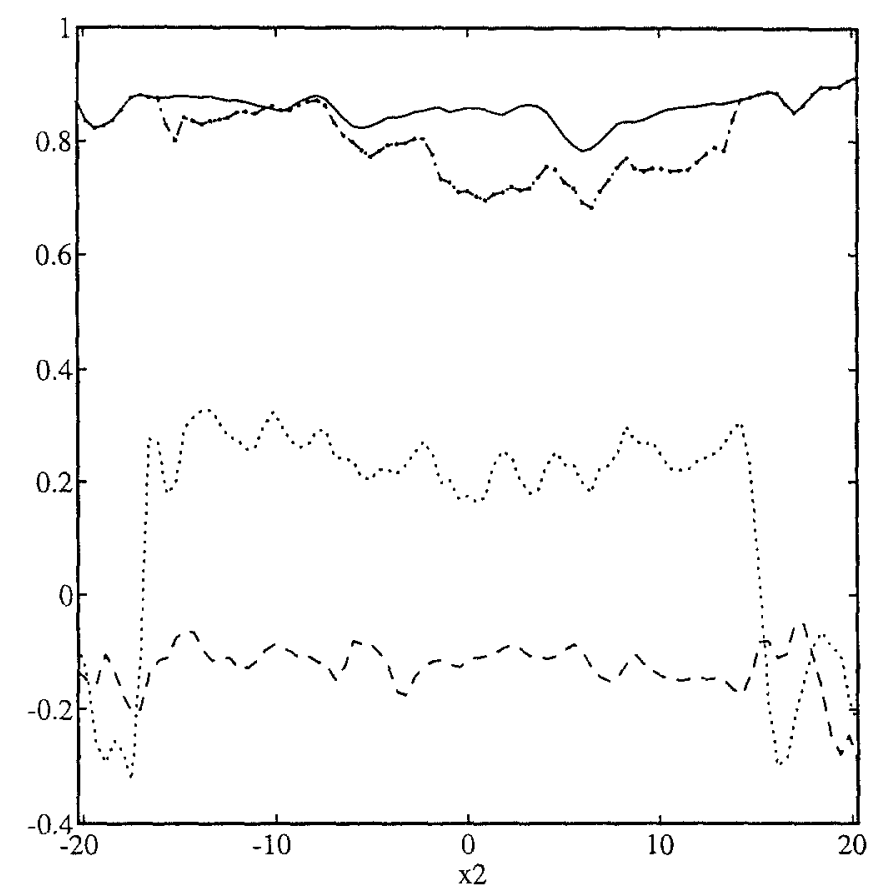

Fig. 1. Correlations with $\partial_{j}\left(\bar{\rho} \tau_{1 j}\right)$ for the similarity model (solid), the dynamic model (dashed), the dynamic model with $C_{I}=0$ (dotted) and the dynamic mixed model (dasheddotted), at $\mathrm{M}=0.2$ and $t=80$.

is obtained, indicating that the spatial structures of the resolved turbulent stress and the exact turbulent stress are in good agreement. Furthermore, we calculated the $L_{2}$-norms of the divergence of the exact and modelled turbulent stress in order to compare their magnitudes. Such a comparison reveals that the similarity model provides predictions which are about three times too low.

Next we consider the dynamic Smagorinsky subgrid-model (usually referred to as 'dynamic model') in which the coefficient of the Smagorinsky model is obtained using Germano's identity (Germano [2]). The dynamic procedure is not restricted to the Smagorinsky model, but is a mechanism to compute variable coefficients for any model which has unknown coefficients. Moin et al. [7] generalised the dynamic model to the compressible case, modelling the isotropic part and the trace-free part separately:

$$
\tau_{i j}^{(2)}=\frac{2}{3} C_{I} \Delta^{2}|\hat{S}|^{2} \delta_{i j}-\nu_{t} \hat{S}_{i j}, \quad \nu_{t}=C_{S} \Delta^{2}|\hat{S}|,
$$

where $|\hat{S}|^{2}=\hat{S}_{i j}^{2} / 2$. The coefficients $C_{I}$ and $C_{S}$, which are functions of time and space, are obtained using the dynamical procedure described in $[2,7]$. Correlations for this model and the simpler model with $C_{I}=0$ are shown in Fig. 1. First, we observe that the model with $C_{I}=0$ gives a better correlation, although for both cases correlations are small. The reason is that the dynamically obtained $C_{I}$ turns 
out to be negative in large parts of the shear layer, which violates realizability conditions (Schumann [21]), using this model with the box filter (Vreman et al. [22]). As a consequence, LES conducted with incorporation of the dynamic model for $C_{I}$ turns out to be unstable for the cases considered in this paper. The norms of the divergences $\left(C_{I}=0\right)$ are approximately equal to those of the exact turbulent stress. Hence, with respect to the magnitude, the dynamic eddy-viscosity model is superior to the similarity model. However, with respect to the correlation with the exact stresses (Fig. 1) and also in actual LES (Section 4; Fig. 3), the similarity model is better than the dynamic eddy-viscosity model.

Recently, Zang et al. [4] introduced the dynamic mixed model:

$$
\tau_{i j}^{(3)}=\tau_{i j}^{(1)}+\tau_{i j}^{(2)} \text {. }
$$

In this case the dynamic model coefficients $C_{S}\left(C_{I}=0\right)$ is obtained by application of the Germano identity to the linear combination of the similarity model and the Smagorinsky model. The model inherits part of the excellent correlation of the similarity model (Fig. 1). However, although the norms of the divergences are higher than for the similarity model, they are still too low. A more consistent formulation of the dynamic mixed model predicts higher norms and gives even better results [23]. This alternative dynamic mixed model is not considered in this paper.

Figure 1 only shows the divergence of the turbulent stress in the first component of the momentum equation at $M=0.2$. Results for the other components and results for the $M=0.6$ case are similar to those shown in Fig. 1 .

\subsection{SUBGRID-MODELS FOR THE ENERGY EQUATION}

Next we turn to the subgrid-terms in the filtered energy equation. First we determine the relative importance of the subgrid-terms by explicitly calculating them using the DNS database. In Fig. 2 the $L_{2}$-norms of these terms in parallel planes with constant $x_{2}$ are plotted for the $M=0.2$ case, showing that these subgrid-terms are of the same order of magnitude for the compressible mixing layer. Notice that this result does not imply that subgrid-modelling in the filtered energy equation is necessary. However, when it is found necessary, these four subgrid-terms should all be modelled, including the usually neglected $\pi$ and $\varepsilon$. From the $a$ priori point of view it is not consistent to model for example $\beta$, while neglecting $\alpha, \pi$ and $\varepsilon$. The $\mathrm{M}=0.2$ and $\mathrm{M}=0.6$ cases do not differ much with respect to the relative order of magnitude of the subgrid-terms. The main difference is that $\varepsilon$ is somewhat larger compared to the other subgrid-terms for the higher Mach number case. This might be due to the influence of dilatational dissipation, a concept which Zeman [10] and Sarkar et al. [11] introduced for $\varepsilon$ in case an ensemble averaging operator is used instead of the spatial filtering operator employed in LES.

In the following we discuss existing and propose new subgrid-models for the subgrid-terms in the filtered energy equation. The term $\alpha$ depends on filtered 


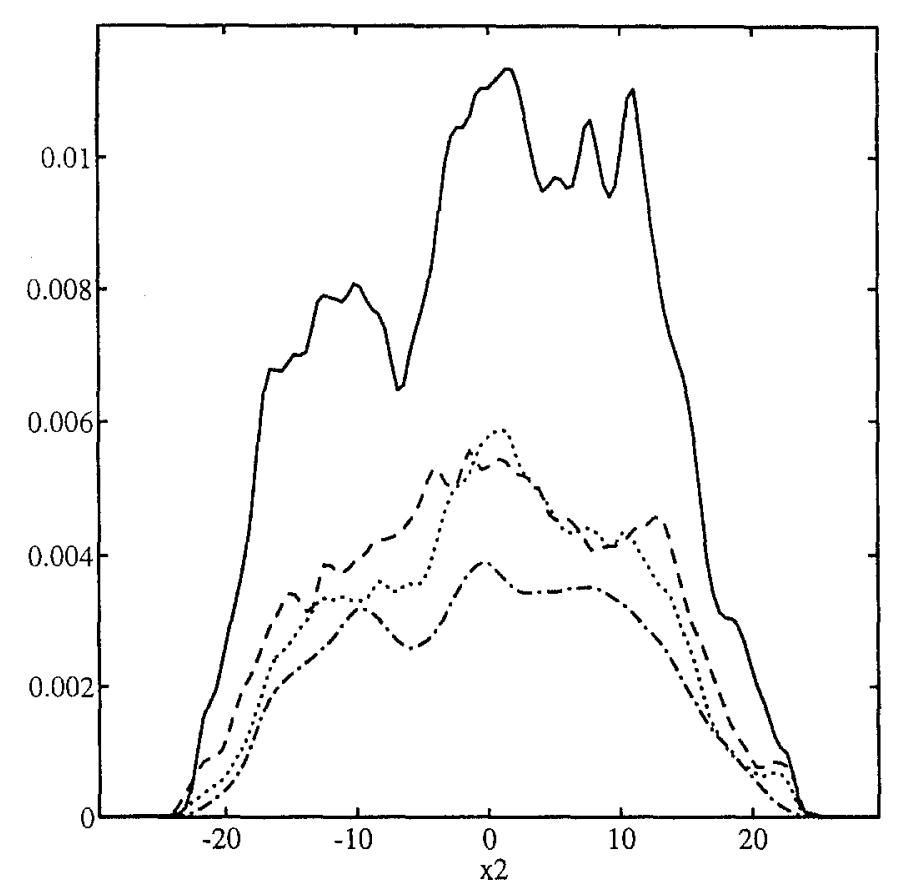

Fig. 2. $L_{2}$-norms for the subgrid-terms in the energy equation; $\alpha$ (solid), $\beta$ (dashed), $\pi$ (dotted) and $\varepsilon$ (dashed-dotted), at $\mathrm{M}=0.2$ and $t=80$.

velocities and the turbulent stress $\tau_{i j}$. Hence, $\alpha$ follows from the model for $\tau_{i j}$. For $\beta$ we consider three models, which are obtained using the concepts introduced for the modelling of $\tau_{i j}$ :

$$
\begin{aligned}
& \beta^{(1)}=\partial_{j}\left(\overline{\bar{p} \tilde{u}_{j}}-\overline{\bar{p}} \tilde{\tilde{u}}_{j}\right) /(\gamma-1), \\
& \beta^{(2)}=-\partial_{j}\left(\frac{\bar{\rho} \nu_{t}}{(\gamma-1) \operatorname{Pr}_{t} \mathrm{M}^{2}} \partial_{j} \tilde{T}\right), \\
& \beta^{(3)}=\beta^{(1)}+\beta^{(2)} .
\end{aligned}
$$

The first model $\beta^{(1)}$ is the similarity model, i.e. the resolved part of $\beta$. The second model uses the eddy-diffusivity hypothesis expressed in the dynamic eddyviscosity obtained with the standard dynamic model. This formulation is simpler than the formulation given by Moin et al. [7]. A dynamic $\operatorname{Pr}_{t}$ is calculated in [7], while here $\operatorname{Pr}_{t}$ is assumed to be constant and only the eddy-viscosity is calculated dynamically. We also consider a dynamic mixed model $\beta^{(3)}$ for this term, which is a generalisation of the standard mixed model for this term (Erlebacher et al. [6]), using the dynamic eddy-viscosity obtained from the dynamic mixed model for the momentum equation. Results of a priori tests for these models are similar to those presented in the previous subsection for the momentum equation. Global 
correlations with the exact subgrid-term are $0.80,0.40$ and 0.75 respectively for the models in equations (17-19). The norm of the similarity model (17) is about a factor three too low, while the norm of the pure dynamic eddy-diffusivity model (18) is approximately correct for $\operatorname{Pr}_{t}=1$ and the norm of the dynamic mixed model (19) is again slightly under-predicted for $\operatorname{Pr}_{t}=1$. This is similar to the results for the momentum equation.

For the pressure-dilatation $\pi$ we propose a similarity model:

$$
\pi^{(1)}=C_{\pi}\left(\overline{\bar{p} \partial_{j} \tilde{u}_{j}}-\overline{\bar{p}} \partial_{j} \tilde{\tilde{u}}_{j}\right)
$$

The global correlation between $\pi^{(1)}$ and $\pi$ is 0.84 . Since the norm of the similarity model under-predicts the magnitude of the exact subgrid-term and we do not have a corresponding eddy-viscosity or eddy-diffusivity as we had for $\tau_{i j}$ and $\beta$, we use a coefficient $C_{\pi}$ in this model. This coefficient is given the value 2.2 in order to give the norm of $\pi^{(1)}$ the correct value. Notice that the model remains Galilean invariant after multiplication with a constant factor. In Large Eddy Simulation $\pi$ is usually neglected, but in studies of the ensemble averaged equations models for $\pi$ have been proposed. An example is the Zeman model [10], which relates $\pi$ to the time derivative of the pressure variance $\overline{p^{2}}-\bar{p}^{2}$. Within the LES approach for the compressible shear layer, this model extremely under-predicts the norm of $\pi$ and yields an almost zero correlation. The poor behaviour is probably due to the local filtering technique employed in LES.

Finally we propose models for the turbulent dissipation rate $\varepsilon$ :

$$
\begin{aligned}
& \varepsilon^{(1)}=C_{1}\left(\overline{\hat{\sigma}_{i j} \partial_{j} \tilde{u}_{i}}-\overline{\hat{\sigma}}_{i j} \partial_{j} \tilde{\tilde{u}}_{i}\right), \\
& \varepsilon^{(2)}=C_{2} \bar{\rho} \frac{k^{3 / 2}}{\Delta}, \quad k=\left(\widetilde{\tilde{u}_{i} \tilde{u}_{i}}-\tilde{\tilde{u}}_{i} \tilde{\tilde{u}}_{i}\right) / 2, \\
& \varepsilon^{(3)}=C_{3} \bar{\rho} \frac{k^{3 / 2}}{\Delta}, \quad k=\sqrt{3 / 4} \nu_{t}|\hat{S}| .
\end{aligned}
$$

The first model employs the similarity idea. The models $\varepsilon^{(2)}$ and $\varepsilon^{(3)}$, which are computationally substantially more efficient than the first model, use a standard approximation for $\varepsilon$ which in the concept of LES is used in e.g. one-equation models for incompressible flow (Wong [24]). The symbol $k$ represents the turbulent kinetic energy, being half the trace of the turbulent stress. In $\varepsilon^{(2)}$ we use the similarity model for $k$, whereas in the third model $k$ is replaced by a theoretical lower-bound using an eddy-viscosity formulation (Vreman et al. [22]). Here $\nu_{t}$ denotes the dynamic eddy-viscosity obtained with the standard dynamic model. It should be observed that the models $\varepsilon^{(2)}$ and $\varepsilon^{(3)}$ require a positive $k$. Only filters with positive filter kernels yield a positive definite $\tau_{i j}$ and, consequently a positive $k$ [22]. The box filter, applied in this paper, satisfies this requirement, in contrast to e.g. the spectral cut-off filter. A priori tests for these three models yield correlations of 
$0.81,0.60$ and 0.58 respectively, while the constants are given the values $C_{1}=8$, $C_{2}=1.6$ and $C_{3}=0.6$ in order to obtain the correct magnitude. In the transitional regime (e.g. at $t=20$ ) these constants are somewhat lower, while estimates for $C_{3}$ are different for other times and Mach numbers.

The dynamic procedure could be used in order to dynamically adjust the constants $\operatorname{Pr}_{t}, C_{\pi}$ and $C_{i}$. However, application of the dynamic procedure to all these models will considerably increase the computational cost of compressible LES. Furthermore, the dynamic procedure for these coefficients is not likely to alter the results of the Large Eddy Simulations at the Mach numbers considered in this paper. For the values given above to $\operatorname{Pr}_{t}, C_{\pi}, C_{1}$ and $C_{2}$, the norms of the subgrid-models were found to match the norms of the corresponding subgrid-terms at most times for both the $\mathrm{M}=0.2$ and $\mathrm{M}=0.6$ cases. However, when high Mach numbers or other flows are considered, other values for the constants $\operatorname{Pr}_{t}, C_{\pi}$ and $C_{i}$ might be required and the dynamic procedure would be a useful mechanism to determine these values.

\section{A Posteriori Tests}

Large Eddy Simulations using the models in the previous section are conducted at $\mathrm{M}=0.2$ and $\mathrm{M}=0.6$ on a grid with $32^{3}$ cells, i.e. the filter width equals twice the grid size. The initial conditions for the DNS are filtered to obtain the initial conditions for LES. The numerical method adopted is as described in the previous section for the DNS, with the addition that the subgrid-terms are discretized in the same way as the viscous terms.

In Fig. 3 the evolutions of the total kinetic energy and momentum thickness are shown for several models at $M=0.2$. It appears that for this Mach number subgrid-modelling in the energy equation is not necessary. We have observed virtually no change of the results shown in these figures when subgrid-models for the subgrid-terms in the energy equation were included, compared to the case that these subgrid-terms were neglected. For the momentum equations results for the similarity model, the standard dynamic model and the dynamic mixed model are shown and compared with the filtered DNS and a coarse grid DNS. For all three models we observe that the results of LES are better than those corresponding to the coarse grid DNS. Moreover, the dynamic mixed model gives better results than the similarity and standard dynamic model. Since the a priori test demonstrated that the magnitude of the turbulent stress predicted by the dynamic mixed model is somewhat too low, we have tested an alternative dynamic mixed model, which is the sum of the similarity model and the standard dynamic model. This model yields results compared to those of the first dynamic mixed model.

In Fig. 4 the total kinetic energy and momentum thickness are shown for the $\mathrm{M}=0.6$ case. In all these simulations the dynamic mixed model is used in the momentum equations. For the energy equation several cases are considered. First, all subgrid-terms are modelled, using $\alpha=\tilde{u}_{i} \partial_{j} \bar{\rho} \tau_{i j}^{(3)}, \beta=\beta^{(3)}, \pi=\pi^{(1)}$ and 

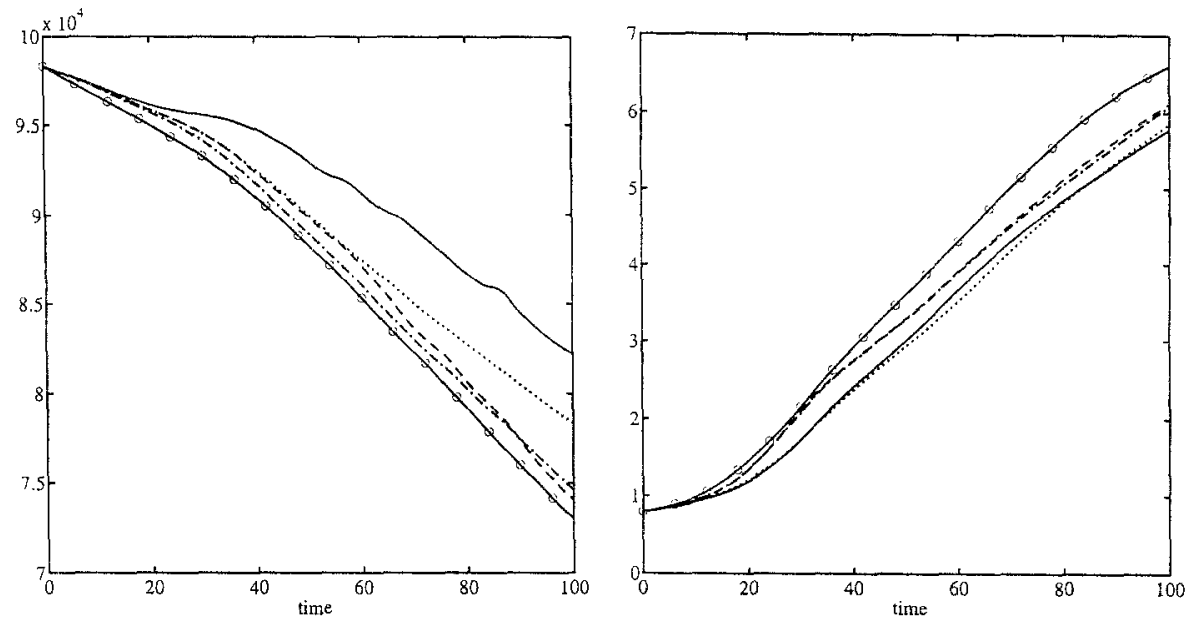

Fig. 3. Total kinetic energy (left) and momentum thickness (right) for the filtered fine-grid DNS at $M=0.2$ (solid with marker ' $o$ '), a coarse-grid DNS (solid) compared with LES using the similarity model (dashed), the dynamic model (dotted) and the dynamic mixed model (dashed-dotted). All subgrid-terms in the energy equation are set zero.
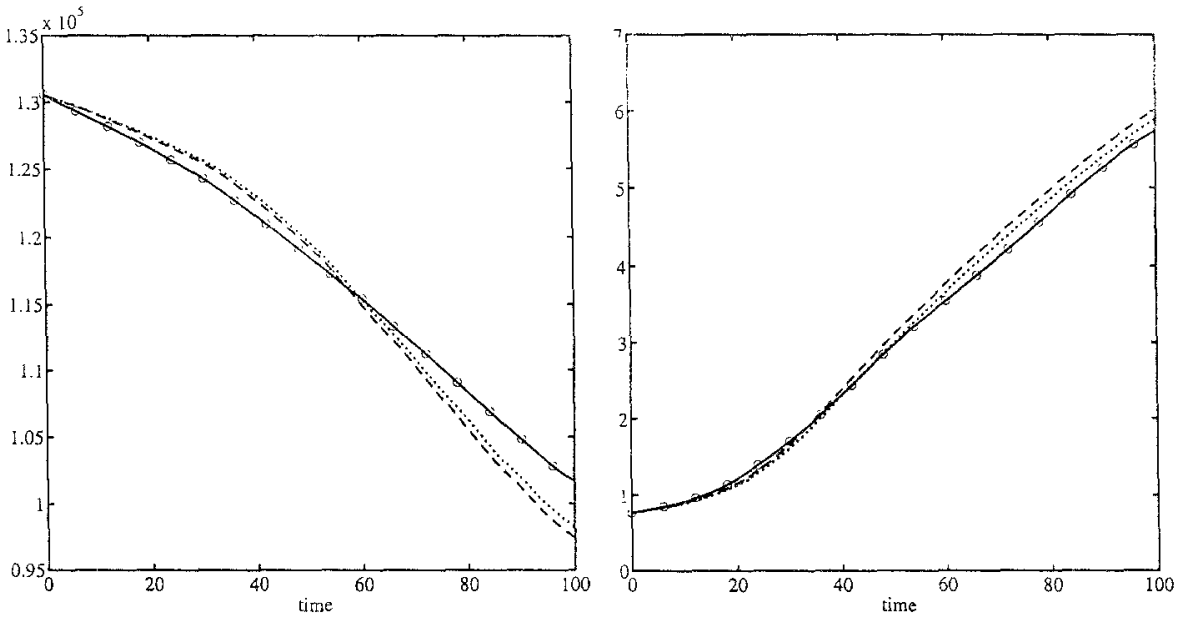

Fig. 4. Total kinetic energy (left) and momentum thickness (right) for the filtered fine-grid DNS at M $=0.6$ (solid with marker ' $o$ ') compared with LES using the dynamic mixed model for the momentum equation in combination with the following modelling assumptions for the energy equation: $\alpha=\beta=\varepsilon=0$ (dashed); $\alpha=\tilde{u}_{i} \partial_{j}\left(\bar{\rho} \tau_{i j}^{(3)}\right), \beta=\beta^{(3)}, \pi=\pi^{(1)}, \varepsilon=\varepsilon^{(2)}$ (dotted).

$\varepsilon=\varepsilon^{(2)}$. In contrast to the $M=0.2$ case, incorporation of subgrid-models for the filtered energy equation yields visible improvement, when compared with a simulation in which no model for the energy equation is employed (see Fig. 4), although also for this Mach number the effect is still small. Other simulations use $\alpha=\beta=\pi=0$ and either $\varepsilon=\varepsilon^{(1)}$ or $\varepsilon=\varepsilon^{(2)}$. The results are virtually identical to those corresponding to the simulation in which all subgrid-terms are retained. 
Although the a priori tests do not reveal $\varepsilon$ to be the dominant subgrid-term, it is the only term in an actual LES which significantly influences the simulation. The fact that $\varepsilon$ is positive everywhere when a box filter is applied (Vreman et al. [22]) could be an explanation for this behaviour. Finally we have performed DNS and LES starting from other initial conditions. These simulations confirm the findings above.

\section{Conclusions}

In this paper the subgrid-modelling problem of compressible LES has been addressed. A priori tests and a posteriori tests were performed for the threedimensional compressible temporal mixing layer at convective Mach numbers of 0.2 and 0.6. As a model for the turbulent stress in the momentum equations the similarity model appears to be as good as the standard dynamic model. The recently developed dynamic mixed model for incompressible flow has now been applied to compressible flow and shown to be superior to the two models mentioned before. Furthermore, the subgrid-terms of the filtered energy equation were considered in detail. A priori tests have shown that the usually neglected pressure-dilatation $(\pi)$ and the turbulent dissipation rate $(\varepsilon)$ are as large as the pressure divergence term $(\beta)$. Subgrid-models for these terms have been proposed: the dynamic mixed model for $\beta$, a similarity model for $\pi$ and a similarity and $k$-dependent model for $\varepsilon$. The coefficients which appear in these models have been estimated using DNS data. To obtain these coefficients with a dynamic procedure as well might improve the models. A posteriori tests indicate that all subgrid-terms in the filtered energy equation may be neglected if the Mach number is low, while at a Mach number of 0.6 the turbulent dissipation rate $\varepsilon$ was found to have the most significant influence. However, the other subgrid-terms in the filtered energy equation might become more important if higher convective Mach numbers are used.

\section{Acknowledgement}

The time for the computations was provided by the Stichting Nationale Computerfaciliteiten (National Computing Facilities Foundation, NCF), which is financially supported by the Nederlandse Organisatie van Wetenschappelijk Onderzoek (Netherlands Organization for Scientific Research, NWO).

\section{References}

1. Rogallo, R.S. and Moin, P., Numerical simulation of turbulent flows. Ann. Rev. Fluid Mech. 16 (1984) 99-137.

2. Germano, M., Turbulence: the filtering approach. J. Fluid Mech. 238 (1992) 325-336.

3. Bardina, J., Ferziger, J.H. and Reynolds, W.C., Improved turbulence models based on LES of homogeneous incompressible turbulent flows. Department of Mechanical Engineering, Report No. TF-19, Stanford (1984).

4. Zang, Y., Street, R.L. and Koseff, J.R., A dynamic mixed subgrid-scale model and its application to turbulent recirculating flows. Phys. Fluids A 5 (1993) 3186-3196. 
5. Yoshizawa, Y., Statistical theory for compressible turbulent shear flows, with the application to subgrid modelling. Phys. Fluids 29 (1986) 2152-2164.

6. Erlebacher, G., Hussaini, M.Y., Speziale, C.G. and Zang, T.A., Toward the Large-Eddy Simulation of compressible turbulent flows. J. Fluid Mech. 238 (1992) 155-185.

7. Moin, P., Squires, K., Cabot, W. and Lee, S., A dynamic subgrid-scale model for compressible turbulence and scalar transport. Phys. Fluids A 3 (1991) 2746-2757.

8. El-Hady, N.M., Zang, T.A. and Piomelli, U., Dynamic subgrid-scale modelling for high-speed transitional boundary layers. ASME Fed-Vol. 162 (1993) 103-112.

9. Blaisdell, G.A., Mansour, N.N. and Reynolds, W.C., Compressibility effects on the growth and structure of homogeneous turbulent shear flow. J. Fluid Mech. 256 (1993) 443-485.

10. Zeman, O., On the decay of compressible isotropic turbulence. Phys. Fluids A 3 (1991) 951-955.

11. Sarkar, S., Erlebacher, G., Hussaini, M.Y. and Kreiss, H.O., The analysis and modelling of dilatational terms in compressible turbulence. J. Fluid Mech. 227 (1991) 473-493.

12. Geurts, B., Vreman, B., Kuerten, H. and Theofilis, V., LES modelling errors in free and wallbounded compressible shear layers. In: Rodi, W. and Martelli, F. (eds), Engineering Turbulence Modelling and Experiments 2. Amsterdam: Elsevier (1993) pp. 325-334.

13. Meneveau, C., Statistics of turbulence subgrid-scale stresses: Necessary conditions and experimental tests. Phys. Fluids 6 (1994) 815-833.

14. Reynolds, W.C., The potential and limitations of direct and large eddy simulations. In: Lumley, J.L. (ed.), Whither Turbulence? Turbulence at Crossroads. Berlin: Springer-Verlag (1990) p. 313.

15. Ragab, S.A. and Wu, J.L., Linear instabilities in two-dimensional compressible mixing layers. Phys. Fluids A 1 (1989) 957-966.

16. Sandham, N.D. and Reynolds, W.C., Three-dimensional simulations of large eddies in the compressible mixing layer. J. Fluid Mech. 224 (1991) 133-158.

17. Comte, P., Lesieur, M. and Lamballais, E., Large and small-scale stirring of vorticity and a passive scalar in a 3D temporal mixing layer. Phys. Fluids A 4 (1992) 2761-2778.

18. Kuerten, J.G.M., Numerical definition document for the ISNaS time-explicit flow solver. Memorandum No. 934, University of Twente (1991).

19. Moser, R.D. and Rogers, M., The three-dimensional evolution of a plane mixing layer: Pairing and transition to turbulence. J. Fluid Mech. 247 (1993) 275-320.

20. Liu, S., Meneveau, C. and Katz, J., Experimental study of similarity subgrid-scale models of turbulence in the far-field of a jet. In: Voke, P.R., Kleiser, L. and Chollet, J.P. (eds), Direct and Large-Eddy Simulation I. Dordrecht: Kluwer Academic Publishers (1994) pp. 37-48.

21. Schumann, U., Modelling of Reynolds-stress turbulence models. Phys. Fluids 20 (1977) 721725.

22. Vreman, B., Geurts, B. and Kuerten, H., Realizability conditions for the turbulent stress tensor in large-eddy simulation. J. Fluid Mech. 278 (1994) 351-362.

23. Vreman, B., Geurts, B. and Kuerten, H., On the formulation of the dynamic mixed subgrid-scale model. Phys. Fluids 6 (1994) 4057-4059.

24. Wong, V.C., A proposed statistical-dynamic closure method for the linear or nonlinear subgridscale stresses. Phys. Fluids A 4 (1992) 1080-1082. 